\title{
Oscillation properties for solutions of a kind of partial fractional differential equations with damping \\ term
}

Wei Nian Li*, Weihong Sheng

Department of Mathematics, Binzhou University, Shandong 256603, PR China.

Communicated by R. Saadati

\section{Abstract}

The aim of the present paper is to obtain sufficient conditions for oscillation of solutions of partial fractional differential equations with the damping term of the form

$$
\begin{aligned}
D_{+, t}^{1+\alpha} u(x, t)+p(t) D_{+, t}^{\alpha} u(x, t)= & a(t) \Delta u(x, t)+\sum_{i=1}^{m} a_{i}(t) \Delta u\left(x, t-\tau_{i}\right) \\
& -q(x, t) \int_{0}^{t}(t-\xi)^{-\alpha} u(x, \xi) d \xi, \quad(x, t) \in \Omega \times \mathbb{R}_{+} \equiv G .
\end{aligned}
$$

Two examples are given to illustrate the main results. (c)2016 All rights reserved.

Keywords: Oscillation, fractional partial differential equation, Riemann-Liouville derivative, damping term.

2010 MSC: 35B05, 35R11, 26A33.

\section{Introduction}

Fractional differential equations are generalizations of classical differential equations to an arbitrary order and have been widely applied in various areas of engineering, physics, mechanics, chemistry, aerodynamics, finance, applied mathematics, bioengineering, nonlinear control and so on. There has been a significant

\footnotetext{
${ }^{*}$ Corresponding author

Email addresses: wnli@263.net (Wei Nian Li), wh-sheng@163.com (Weihong Sheng)
} 
development in fractional differential equations in recent years. For example, see the monographs [1, 4, 7, 9, 14.

Recently, the research on the theory of partial fractional differential equations is a very interesting topic and some results are established. Jafari et al. [6] presented the iterative Laplace transform method to derive exact and approximate analytical solutions of fractional partial differential equations. Chen and Jiang [2] exploited the techniques of Lie group analysis to solve $n$-order linear fractional partial differential equation and nonlinear fractional reaction diffusion convection equation. Zheng [12] applied the $\left(G^{\prime} / G\right)$ method to seek exact solutions for several fractional partial differential equations including the space-time fractional $(2+1)$ dimensional dispersive long wave equations, the $(2+1)$-dimensional space time fractional NizhnikNovikov-Veselov system and the time fractional fifth-order Sawada-Kotera equation. Based on a fractional complex transformation, Zheng and Feng[13] obtained Jacobi elliptic function solutions to fractional partial differential equations.

At the same time, a few papers [5, 8, 10, 11] studied the oscillation of fractional partial differential equations which involve the Riemann-Liouville fractional partial derivative. In [11], Prakash et al. considered the oscillation of the fractional partial differential equation

$$
\frac{\partial}{\partial t}\left(r(t) D_{+, t}^{\alpha} u(x, t)\right)+q(x, t) f\left(\int_{0}^{t}(t-\nu)^{-\alpha} u(x, \nu) d \nu\right)=a(t) \Delta u(x, t),
$$

where $(x, t) \in \Omega \times \mathbb{R}_{+} \equiv G$.

Harikrishnan et al. [5] studied the oscillatory behavior of the fractional partial differential equation of the form

$$
D_{+, t}^{\alpha}\left(r(t) D_{+, t}^{\alpha} u(x, t)\right)+q(x, t) f(u(x, t))=a(t) \Delta u(x, t)+g(x, t), \quad(x, t) \in G .
$$

Prakash et al.[10] established the oscillation of a nonlinear fractional partial differential equation with damping and forced term of the form

$$
D_{+, t}^{\alpha}\left(r(t) D_{+, t}^{\alpha} u(x, t)\right)+p(t) D_{+, t}^{\alpha} u(x, t)+q(x, t) f(u(x, t))=a(t) \Delta u(x, t)+g(x, t), \quad(x, t) \in G .
$$

In [8], Li investigated the forced oscillation of fractional partial differential equations with the damping term of the form

$$
\frac{\partial}{\partial t}\left(D_{+, t}^{\alpha} u(x, t)\right)+p(t) D_{+, t}^{\alpha} u(x, t)=a(t) \Delta u(x, t)-q(x, t) u(x, t)+f(x, t), \quad(x, t) \in G .
$$

\section{Formulation of the Problems}

In this paper, we are concerned with establishing criteria for oscillation of solutions of partial fractional differential equations with the damping term of the form

$$
\begin{aligned}
D_{+, t}^{1+\alpha} u(x, t)+p(t) D_{+, t}^{\alpha} u(x, t)= & a(t) \Delta u(x, t)+\sum_{i=1}^{m} a_{i}(t) \Delta u\left(x, t-\tau_{i}\right) \\
& -q(x, t) \int_{0}^{t}(t-\xi)^{-\alpha} u(x, \xi) d \xi,(x, t) \in \Omega \times \mathbb{R}_{+} \equiv G,
\end{aligned}
$$

where $\Omega$ is a bounded domain in $\mathbb{R}^{n}$ with a piecewise smooth boundary $\partial \Omega, \alpha \in(0,1)$ is a constant and $D_{+, t}^{\alpha} u(x, t)$ is the Riemann-Liouville fractional derivative of order $\alpha$ of $u$ with respect to $t, \Delta$ is the Laplacian in $\mathbb{R}^{n}$.

We assume throughout this paper that

(A1) $a \in C([0, \infty) ;[0, \infty)), p \in C([0, \infty) ;[0, \infty)), a_{i} \in C([0, \infty) ;[0, \infty))$ and $\tau_{i} \geq 0$ is a constant, $i=$ $1,2, \cdots, m$;

(A2) $q \in C\left(\bar{G} ; \mathbb{R}_{+}\right)$and $q(t)=\min _{x \in \bar{\Omega}} q(x, t)$.

Consider one of the two following boundary conditions 


$$
\frac{\partial u(x, t)}{\partial N}+g(x, t) u(x, t)=0,(x, t) \in \partial \Omega \times \mathbb{R}_{+},
$$

or

$$
u(x, t)=0,(x, t) \in \partial \Omega \times \mathbb{R}_{+},
$$

where $N$ is the unit exterior normal vector to $\partial \Omega$ and $g(x, t)$ is a nonnegative continuous function on $\partial \Omega \times \mathbb{R}_{+}$.

By a solution of the problem (2.1)-(2.2) (or (2.1)-(2.3)), we mean a function $u(x, t)$ which satisfies $(2.1)$ on $\bar{G}$ and the boundary condition $(2.2)$ (or 2.3$)$ ).

A solution $u(x, t)$ of the problem (2.1)-2.2) (or 2.1)-2.3) is said to be oscillatory in $G$ if it is neither eventually positive nor eventually negative, otherwise it is non-oscillatory.

Definition 2.1 ([7]). The Riemann-Liouville fractional integral of order $\alpha>0$ of a function $y: \mathbb{R}_{+} \rightarrow \mathbb{R}$ on the half-axis $\mathbb{R}_{+}$is given by

$$
I_{+}^{\alpha} y(t):=\frac{1}{\Gamma(\alpha)} \int_{0}^{t}(t-\xi)^{\alpha-1} y(\xi) d \xi \quad \text { for } \quad t>0
$$

provided the right hand side is pointwise defined on $\mathbb{R}_{+}$, where $\Gamma$ is the gamma function.

Definition 2.2 ([7]). The Riemann-Liouville fractional derivative of order $\alpha>0$ of a function $y: \mathbb{R}_{+} \rightarrow \mathbb{R}$ on the half-axis $\mathbb{R}_{+}$is given by

$$
\begin{aligned}
D_{+}^{\alpha} y(t) & :=\frac{d^{\lceil\alpha\rceil}}{d t^{\lceil\alpha\rceil}}\left(I_{+}^{\lceil\alpha\rceil-\alpha} y\right)(t) \\
& =\frac{1}{\Gamma(\lceil\alpha\rceil-\alpha)} \frac{d^{\lceil\alpha\rceil}}{d t^{\lceil\alpha\rceil}} \int_{0}^{t}(t-\xi)^{\lceil\alpha\rceil-\alpha-1} y(\xi) d \xi \text { for } t>0
\end{aligned}
$$

provided the right hand side is pointwise defined on $\mathbb{R}_{+}$, where $\lceil\alpha\rceil$ is the ceiling function of $\alpha$.

Definition 2.3 ([7]). The Riemann-Liouville partial fractional derivative of order $0<\alpha<1$ with respect to $t$ of a function $u(x, t)$ is given by

$$
D_{+, t}^{\alpha} u(x, t):=\frac{1}{\Gamma(1-\alpha)} \frac{\partial}{\partial t} \int_{0}^{t}(t-\xi)^{-\alpha} u(x, \xi) d \xi
$$

provided the right hand side is pointwise defined on $\mathbb{R}_{+}$.

The following lemmas are very useful in the proof of our main results.

Lemma 2.4 ([7]). Let $\alpha \geq 0, m \in \mathbb{N}$ and $D=\frac{d}{d t}$. If the fractional derivatives $D_{+}^{\alpha} y(t)$ and $D_{+}^{\alpha+m} y(t)$ exist, then

$$
D^{m}\left(D_{+}^{\alpha} y(t)\right)=D_{+}^{\alpha+m} y(t)
$$

Lemma 2.5 ([1]). Let

$$
\widetilde{E}(t)=: \int_{0}^{t}(t-\xi)^{-\alpha} y(\xi) d \xi \quad \text { for } \quad \alpha \in(0,1) \quad \text { and } \quad t>0 .
$$

Then $\widetilde{E}^{\prime}(t)=\Gamma(1-\alpha) D_{+}^{\alpha} y(t)$. 


\section{Oscillation of the Problem (2.1) -2.2}

Theorem 3.1. Assume that for some $t_{0}>0$,

$$
\int_{t_{0}}^{\infty} \exp \left(-\int_{t_{0}}^{t} p(\xi) d \xi\right) d t=\infty
$$

and

$$
\int_{t_{0}}^{\infty} q(s) v(s) d s=\infty
$$

where

$$
v(t)=\exp \left(\int_{t_{0}}^{t} p(s) d s\right), t \geq t_{0} .
$$

Then every solution of the problem (2.1) -2.2 is oscillatory in $G$.

Proof. Suppose to the contrary that there is a nonoscillatory solution $u(x, t)$ to the problem 2.1 - 2.2 which has no zero in $\Omega \times\left[t_{0}, \infty\right)$ for some $t_{0}>0$. Without loss of generality, we may assume that $u(x, t)>$ 0 and $u\left(x, t-\tau_{i}\right)>0$ in $\Omega \times\left[t_{1}, \infty\right)$ for $t_{1} \geq t_{0}, i=1,2, \cdots, m$.

Integrating 2.1 with respect to $x$ over the domain $\Omega$, we have

$$
\begin{aligned}
\int_{\Omega} D_{+, t}^{1+\alpha} u(x, t) d x+p(t) \int_{\Omega} D_{+, t}^{\alpha} u(x, t) d x= & a(t) \int_{\Omega} \Delta u(x, t) d x+\sum_{i=1}^{m} \int_{\Omega} a_{i}(t) \Delta u\left(x, t-\tau_{i}\right) d x \\
& -\int_{\Omega} q(x, t)\left(\int_{0}^{t}(t-\xi)^{-\alpha} u(x, \xi) d \xi\right) d x, t \geq t_{1} .
\end{aligned}
$$

Green's formula and $(2.2)$ yield

$$
\int_{\Omega} \Delta u(x, t) d x=\int_{\partial \Omega} \frac{\partial u(x, t)}{\partial N} d S=-\int_{\partial \Omega} g(x, t) u(x, t) d S \leq 0, \quad t \geq t_{1}
$$

and

$$
\begin{aligned}
\int_{\Omega} \Delta u\left(x, t-\tau_{i}\right) d x & =\int_{\partial \Omega} \frac{\partial u\left(x, t-\tau_{i}\right)}{\partial N} d S \\
& =-\int_{\partial \Omega} g\left(x, t-\tau_{i}\right) u\left(x, t-\tau_{i}\right) d S \leq 0, t \geq t_{1}, i=1,2, \cdots, m,
\end{aligned}
$$

where $d S$ is the surface element on $\partial \Omega$. Noting the assumpition (A2), we have

$$
\begin{aligned}
\int_{\Omega} q(x, t)\left(\int_{0}^{t}(t-\xi)^{-\alpha} u(x, \xi) d \xi\right) d x & \geq q(t) \int_{\Omega}\left(\int_{0}^{t}(t-\xi)^{-\alpha} u(x, \xi) d \xi\right) d x \\
& =q(t) \int_{0}^{t}(t-\xi)^{-\alpha}\left(\int_{\Omega} u(x, \xi) d x\right) d \xi, \quad t \geq t_{1} .
\end{aligned}
$$

Let

$$
V(t)=\int_{\Omega} u(x, t) d x
$$

Combining (3.4)-(3.7), we obtain

$$
D_{+}^{1+\alpha} V(t)+p(t) D_{+}^{\alpha} V(t) \leq-q(t) E(t), \quad t \geq t_{1},
$$

where

$$
E(t)=\int_{0}^{t}(t-\xi)^{-\alpha} V(\xi) d \xi>0
$$


Using Lemma 2.4, it follows from (3.3), (3.8) and (3.9) that

$$
\begin{aligned}
\left(\left(D_{+}^{\alpha} V(t)\right) v(t)\right)^{\prime} & =\left(D_{+}^{1+\alpha} V(t)\right) v(t)+\left(D_{+}^{\alpha} V(t)\right) p(t) v(t) \\
& \leq-q(t) E(t) v(t)<0, t \geq t_{1} .
\end{aligned}
$$

Hence, $\left(D_{+}^{\alpha} V(t)\right) v(t)$ is strictly decreasing in $\left[t_{1}, \infty\right)$ and we can claim that $D_{+}^{\alpha} V(t)>0$ for $t \geq t_{1}$. In fact, if $D_{+}^{\alpha} V(t) \leq 0$ for $t \geq t_{1}$, then there exists a $T \geq t_{1}$ such that

$$
\left(D_{+}^{\alpha} V(t)\right) v(t)<\left(D_{+}^{\alpha} V(T)\right) v(T)=C_{1}<0, t>T .
$$

By Lemma 2.5, from 3.11 we have

$$
\frac{E^{\prime}(t)}{\Gamma(1-\alpha)}=D_{+}^{\alpha} V(t)<\frac{C_{1}}{v(t)}=C_{1} \exp \left(-\int_{t_{0}}^{t} p(s) d s\right), t \geq T .
$$

Integrating (3.12) from $T$ to $t$, we obtain

$$
E(t)<E(T)+\Gamma(1-\alpha) C_{1} \int_{T}^{t} \exp \left(-\int_{t_{0}}^{t} p(\xi) d \xi\right) d s, t>T .
$$

Letting $t \rightarrow \infty$ in $(3.13)$, we have $\lim _{t \rightarrow \infty} E(t)=-\infty$, which contradicts with the fact $E(t)>0$.

Define the function

$$
w(t)=\frac{\left(D_{+}^{\alpha} V(t)\right) v(t)}{E(t)}, t \geq t_{1} .
$$

Then $w(t)>0$ and

$$
\begin{aligned}
w^{\prime}(t) & =\frac{\left[\left(D_{+}^{\alpha} V(t)\right) v(t)\right]^{\prime}}{E(t)}-\frac{\left(D_{+}^{\alpha} V(t)\right) v(t) E^{\prime}(t)}{E^{2}(t)} \\
& =\frac{\left(D_{+}^{1+\alpha} V(t)\right) v(t)+\left(D_{+}^{\alpha} V(t)\right) p(t) v(t)}{E(t)}-\frac{\Gamma(1-\alpha)\left(D_{+}^{\alpha} V(t)\right)^{2} v(t)}{E^{2}(t)} \\
& \leq-q(t) v(t), t \geq t_{1} .
\end{aligned}
$$

Integrating (3.15) from $t_{1}$ to $t$, we obtain

$$
w(t) \leq w\left(t_{1}\right)-\int_{t_{1}}^{t} q(s) v(s) d s .
$$

Letting $t \rightarrow \infty$ in (3.16) and noting the assumption (3.2), we can obtain a contradiction with $w(t)>0$. The proof of Theorem 3.1 is complete.

Theorem 3.2. Assume that (3.1) holds and

$$
\int_{t_{0}}^{\infty}\left[q(s)-\frac{p^{2}(s)}{4 \Gamma(1-\alpha)}\right] d s=\infty, t_{0}>0
$$

Then every solution of the problem (2.1) -2.2 is oscillatory in $G$.

Proof. Suppose to the contrary that there is a nonoscillatory solution $u(x, t)$ to the problem (2.1)-(2.2) which has no zero in $\Omega \times\left[t_{0}, \infty\right)$ for some $t_{0}>0$. Without loss of generality, we may assume that $u(x, t)>$ 0 and $u\left(x, t-\tau_{i}\right)>0$ in $\Omega \times\left[t_{1}, \infty\right)$ for $t_{1} \geq t_{0}, i=1,2, \cdots, m$.

As in the proof of Theorem 3.1. we obtain that (3.7) and $D_{+}^{\alpha} V(t)>0$ for $t \geq t_{1}$ hold. Defined the function

$$
\widetilde{w}(t)=\frac{D_{+}^{\alpha} V(t)}{E(t)}, t \geq t_{1}
$$


Then $\widetilde{w}(t)>0$ and

$$
\begin{aligned}
\widetilde{w}^{\prime}(t) & =\frac{D_{+}^{1+\alpha} V(t)}{E(t)}-\frac{E^{\prime}(t) D_{+}^{\alpha} V(t)}{E^{2}(t)} \\
& \leq \frac{-p(t) D_{+}^{\alpha} V(t)-q(t) E(t)}{E(t)}-\frac{\Gamma(1-\alpha)\left(D_{+}^{\alpha} V(t)\right)^{2}}{E^{2}(t)} \\
& =-q(t)-p(t) \widetilde{w}(t)-\Gamma(1-\alpha) \widetilde{w}^{2}(t) \\
& =-q(t)-\left[\sqrt{\Gamma(1-\alpha)} \widetilde{w}(t)+\frac{p(t)}{2 \sqrt{\Gamma(1-\alpha)}}\right]^{2}+\frac{p^{2}(t)}{4 \Gamma(1-\alpha)} \\
& \leq-\left[q(t)-\frac{p^{2}(t)}{4 \Gamma(1-\alpha)}\right], t \geq t_{1} .
\end{aligned}
$$

Integrating (3.19) from $t_{1}$ to $t$, we obtain

$$
\widetilde{w}(t) \leq \widetilde{w}\left(t_{1}\right)-\int_{t_{1}}^{t}\left[q(s)-\frac{p^{2}(s)}{4 \Gamma(1-\alpha)}\right] d s .
$$

Letting $t \rightarrow \infty$ in $(3.20)$, we can obtain a contradiction with $\widetilde{w}(t)>0$. This completes the proof of Theorem 3.2 .

\section{Oscillation of the Problem (2.1) $-(2.3)$}

The following fact [3] will be used.

The smallest eigenvalue $\beta_{0}$ of the Dirichlet problem

$$
\left\{\begin{array}{l}
\Delta \omega(x)+\beta \omega(x)=0, \text { in } \Omega, \\
\omega(x)=0, \text { on } \partial \Omega,
\end{array}\right.
$$

is positive and the corresponding eigenfunction $\varphi(x)$ is positive in $\Omega$.

Theorem 4.1. Assume that the conditions of Theorem 3.1 hold. Then every solution of the problem (2.1)(2.3) is oscillatory in $G$.

Proof. Suppose to the contrary that there is a nonoscillatory solution $u(x, t)$ to the problem (2.1)-(2.3) which has no zero in $\Omega \times\left[t_{0}, \infty\right)$ for some $t_{0}>0$. Without loss of generality, we may assume that $u(x, t)>$ 0 and $u\left(x, t-\tau_{i}\right)>0$ in $\Omega \times\left[t_{1}, \infty\right)$ for $t_{1} \geq t_{0}, i=1,2, \cdots, m$.

Multiplying both sides of $(2.1)$ by $\varphi(x)$ and integrating with respect to $x$ over the domain $\Omega$, we have

$$
\begin{aligned}
\int_{\Omega} D_{+, t}^{1+\alpha} u(x, t) \varphi(x) d x & +p(t) \int_{\Omega} D_{+, t}^{\alpha} u(x, t) \varphi(x) d x \\
= & a(t) \int_{\Omega} \Delta u(x, t) \varphi(x) d x+\sum_{i=1}^{m} \int_{\Omega} a_{i}(t) \Delta u\left(x, t-\tau_{i}\right) \varphi(x) d x \\
& -\int_{\Omega} q(x, t)\left(\int_{0}^{t}(t-\xi)^{-\alpha} u(x, \xi) d \xi\right) \varphi(x) d x, \quad t \geq t_{1} .
\end{aligned}
$$

Green's formula and (2.3) yield

$$
\int_{\Omega} \Delta u(x, t) \varphi(x) d x=\int_{\Omega} u(x, t) \Delta \varphi(x) d x=-\beta_{0} \int_{\Omega} u(x, t) \varphi(x) d x \leq 0, \quad t \geq t_{1},
$$

and

$$
\begin{aligned}
\int_{\Omega} \Delta u\left(x, t-\tau_{i}\right) \varphi(x) d x & =\int_{\Omega} u\left(x, t-\tau_{i}\right) \Delta \varphi(x) d x \\
& =-\beta_{0} \int_{\Omega} u\left(x, t-\tau_{i}\right) \varphi(x) d x \leq 0, \quad t \geq t_{1}, \quad i=1,2, \cdots, m
\end{aligned}
$$


It follows from the assumpition (A2) that

$$
\begin{aligned}
\int_{\Omega} q(x, t)\left(\int_{0}^{t}(t-\xi)^{-\alpha} u(x, \xi) d \xi\right) \varphi(x) d x & \geq q(t) \int_{\Omega}\left(\int_{0}^{t}(t-\xi)^{-\alpha} u(x, \xi) d \xi\right) \varphi(x) d x \\
& =q(t) \int_{0}^{t}(t-\xi)^{-\alpha}\left(\int_{\Omega} u(x, \xi) \varphi(x) d x\right) d \xi, \quad t \geq t_{1} .
\end{aligned}
$$

Let

$$
U(t)=\int_{\Omega} u(x, t) \varphi(x) d x, t \geq t_{1}
$$

Combining (4.1)-(4.4), we have

$$
D_{+}^{1+\alpha} U(t)+p(t) D_{+}^{\alpha} U(t) \leq-q(t) \bar{E}(t), \quad t \geq t_{1},
$$

where

$$
\bar{E}(t)=\int_{0}^{t}(t-\xi)^{-\alpha} U(\xi) d \xi>0 .
$$

Using Lemma 2.4, it follows from (4.5) and (4.6) that

$$
\begin{aligned}
\left(\left(D_{+}^{\alpha} U(t)\right) v(t)\right)^{\prime} & =\left(D_{+}^{1+\alpha} U(t)\right) v(t)+\left(D_{+}^{\alpha} U(t)\right) p(t) v(t) \\
& \leq-q(t) \bar{E}(t) v(t)<0, t \geq t_{1} .
\end{aligned}
$$

Hence, $\left(D_{+}^{\alpha} U(t)\right) v(t)$ is strictly decreasing in $\left[t_{1}, \infty\right)$ and $D_{+}^{\alpha} U(t)>0$ for $t \geq t_{1}$.

The remainder of the proof is similar to that of Theorem 3.1 and we omit it here. The proof of Theorem 4.1 is complete.

It is not difficult to prove the following theorem.

Theorem 4.2. Assume that the conditions of Theorem 3.2 hold. Then every solution of the problem 2.1)(2.3) is oscillatory in $G$.

\section{Examples}

In this section, we give two examples to illustrate our main results.

Example 5.1. Consider the following partial fractional differential equation

$$
\begin{aligned}
D_{+}^{\frac{5}{4}} u(x, t)+\frac{1}{t} D_{+, t}^{\frac{1}{4}} u(x, t)= & \frac{1}{8} e^{t} \Delta u(x, t)+2 t \Delta u(x, t-1) \\
& -\left(x^{3}+\frac{1}{t^{2}}\right) \int_{0}^{t}(t-\xi)^{-\frac{1}{4}} u(x, \xi) d \xi, \quad(x, t) \in(0, \pi) \times \mathbb{R}_{+},
\end{aligned}
$$

with the boundary condition

$$
u_{x}(0, t)=u_{x}(\pi, t)=0, t>0 .
$$

Here $\alpha=\frac{1}{4}, \Omega=(0, \pi), n=1, m=1, a_{1}(t)=2 t, \tau_{1}=1, p(t)=\frac{1}{t}, a(t)=\frac{1}{8} e^{t}$ and $q(x, t)=x^{3}+\frac{1}{t^{2}}$. Hence $q(t)=\frac{1}{t^{2}}$. It is easy to see that

$$
\begin{gathered}
\int_{t_{0}}^{\infty} \exp \left(-\int_{t_{0}}^{t} p(\xi) d \xi\right) d t=\int_{t_{0}}^{\infty} \exp \left(-\int_{t_{0}}^{t} \frac{1}{\xi} d \xi\right) d t=\int_{t_{0}}^{\infty} \frac{t_{0}}{t} d t=\infty \\
v(t)=\exp \left(\int_{t_{0}}^{t} p(\xi) d \xi\right)=\exp \left(\int_{t_{0}}^{t} \frac{1}{\xi} d \xi\right)=\frac{t}{t_{0}}
\end{gathered}
$$


and

$$
\int_{t_{0}}^{\infty} q(s) v(s)=\int_{t_{0}}^{\infty} \frac{1}{s^{2}} \frac{s}{t_{0}} d s=\int_{t_{0}}^{\infty} \frac{1}{t_{0} s} d s=\infty, t_{0}>0 .
$$

Therefore, the conditions of Theorem 3.1 are fulfilled. Then every solution of the problem (5.1) oscillates in $(0, \pi) \times \mathbb{R}_{+}$.

Example 5.2. Consider the following partial fractional differential equation

$$
\begin{aligned}
D_{+}^{\frac{6}{5}} u(x, t)+\frac{1}{t} D_{+, t}^{\frac{1}{5}} u(x, t)= & 3 e^{-t} \Delta u(x, t)+\frac{t}{3} \Delta\left(x, t-\frac{1}{3}\right) \\
& -\left(2 x^{2}+\frac{1}{t}\right) \int_{0}^{t}(t-\xi)^{-\frac{1}{5}} u(x, \xi) d \xi, \quad(x, t) \in(0, \pi) \times \mathbb{R}_{+},
\end{aligned}
$$

with the boundary condition

$$
u(0, t)=u(\pi, t)=0, t>0 .
$$

Here $\alpha=\frac{1}{5}, \Omega=(0, \pi), n=1, m=1, p(t)=\frac{1}{t}, a(t)=3 e^{-t}, a_{1}(t)=\frac{t}{3}, \tau_{1}=\frac{1}{3}$ and $q(x, t)=2 x^{2}+\frac{1}{t}$. Hence $q(t)=\frac{1}{t}$.

We easily see that

$$
\int_{t_{0}}^{\infty} \exp \left(-\int_{t_{0}}^{t} p(\xi) d \xi\right) d t=\int_{t_{0}}^{\infty} \exp \left(-\int_{t_{0}}^{t} \frac{1}{\xi} d \xi\right) d t=\int_{t_{0}}^{\infty} \frac{t_{0}}{t} d t=\infty
$$

and

$$
\int_{t_{0}}^{\infty}\left[q(s)-\frac{p^{2}(s)}{4 \Gamma\left(\frac{4}{5}\right)}\right] d s=\int_{t_{0}}^{\infty}\left[\frac{1}{s}-\frac{1}{4 \Gamma\left(\frac{4}{5}\right) s^{2}}\right] d s=\infty, t_{0}>0 .
$$

Therefore, the conditions of Theorem 4.2 are satisfied. Then every solution of the problem (5.6)-(5.7) oscillates in $(0, \pi) \times \mathbb{R}_{+}$.

\section{Acknowledgements}

This work is supported by the National Natural Science Foundation of China (10971018). The authors thank the referee very much for his valuable comments and suggestions on this paper.

\section{References}

[1] S. Abbas, M. Benchohra, G. M. N'Guérékata, Topics in Fractional Differential Equations, Springer, New York, (2012). 1

[2] C. Chen, Y. L. Jiang, Lie group analysis method for two classes of fractional partial differential equations, Commun. Nonlinear Sci. Numer. Simul., 26 (2015), 24-35. 1

[3] R. Courant, D. Hilbert, Methods of Mathematical Physics, Interscience, New York, (1966). 4

[4] S. Das, Functional Fractional Calculus for System Identification and Controls, Springer, Berlin, (2008). 1

[5] S. Harikrishnan, P. Prakash, J. J. Nieto, Forced oscillation of solutions of a nonlinear fractional partial differential equation, Appl. Math. Comput., 254 (2015), 14-19. 1, 1

[6] H. Jafari, M. Nazari, D. Baleanu, C. M. Khalique, A new approach for solving a system of fractional partial differential equations, Comput. Math. Appl., 66 (2013), 838-843. 1

[7] A. A. Kilbas, H. M. Srivastava, J. J. Trujillo, Theory and Applications of Fractional Differential Equations, Elsevier Science B. V., Amsterdam, (2006). 1, 2.1, 2.2, 2.3, 2.4

[8] W. N. Li, Forced oscillation criteria for a class of fractional partial differential equations with damping term, Math. Probl. Eng., 2015 (2015), 6 pages. 1, 1

[9] I. Podlubny, Fractional Differential Equations, Academic Press Inc., San Diego, (1999). 1

[10] P. Prakash, S. Harikrishnan, M. Benchohra, Oscillation of certain nonlinear fractional partial differential equation with damping term, Appl. Math. Lett., 43 (2015), 72-79. 1,1

[11] P. Prakash, S. Harikrishnan, J. J. Nieto, J. H. Kim, Oscillation of a time fractional partial differential equation, Electron, J. Qual. Theory Differ. Equ., 2014 (2014), 10 pages. 1]2.5 
[12] B. Zheng, Exact solutions for some fractional partial differential equations by the $\left(G^{\prime} / G\right)$ method, Math. Probl. Eng., 2013 (2013), 13 pages. 1

[13] B. Zheng, Q. Feng, A new approach for solving fractional partial differential equations in the sense of the modified Riemann-Liouville derivative, Math. Probl. Eng., 2014 (2014), 7 pages. 1

[14] Y. Zhou, Basic Theory of Fractional Differential Equations, World Scientific Publishing Co. Pte. Ltd., Hackensack, (2014). 1 . 